Research Article

\title{
Wave Motion Analysis in Plane via Hermitian Cubic Spline Wavelet Finite Element Method
}

\author{
Xiaofeng Xue $\mathbb{D}$, Xinhai Wang, Zhen Wang, and Wei Xue \\ Mechanical and Electrical Engineering Department, Yuncheng University, Yuncheng 044000, China \\ Correspondence should be addressed to Xiaofeng Xue; 13892825438@163.com
}

Received 21 February 2020; Revised 5 May 2020; Accepted 11 May 2020; Published 26 August 2020

Academic Editor: Yongteng Zhong

Copyright $\odot 2020$ Xiaofeng Xue et al. This is an open access article distributed under the Creative Commons Attribution License, which permits unrestricted use, distribution, and reproduction in any medium, provided the original work is properly cited.

\begin{abstract}
A plane Hermitian wavelet finite element method is presented in this paper. Wave motion can be used to analyze plane structures with small defects such as cracks and obtain results. By using the tensor product of modified Hermitian wavelet shape functions, the plane Hermitian wavelet shape functions are constructed. Scale functions of Hermitian wavelet shape functions can replace the polynomial shape functions to construct new wavelet plane elements. As the scale of the shape functions increases, the precision of the new wavelet plane element will be improved. The new Hermitian wavelet finite element method which can be used to simulate wave motion analysis can reveal the law of the wave motion in plane. By using the results of transmitted and reflected wave motion, the cracks can be easily identified in plane. The results show that the new Hermitian plane wavelet finite element method can use the fewer elements to simulate the plane structure effectively and accurately and detect the cracks in plane.
\end{abstract}

\section{Introduction}

The application of plate structure in industry is very important [1]. Plane wave motion analysis has been researched in mechanical engineering in recent years [2]. Wave motion analysis remains an open research field in real engineering though mathematical principles are well developed [3]. Many numerical methods are used to analyze the motion of elastic waves area [4]. Manktelow et al. [5] proposed a perturbation analysis by using discretization finite element method to research wave motion in continuous and periodic structures. Wang and Sett [6] used the stochastic Galerkin method, where material parameters and mechanical functions are uncertain to solve the solid mechanics partial differential equation. Gravenkamp et al. [7] resolved the results of high frequencies wave motion by using the following methods: scaled boundary finite element method and the nonuniform rational B-splines. Komijani and Gracie [8] proposed the global enrichment method which adopts the harmonic functions. The merit of global enrichment and the generalized finite element method is that they can analyze wave motion in plane with cracks by using the phantom node method. Pamel et al. [9] derive the finite element equations which can solve the problem of three-dimensional elastic dynamic wave scattering motion. The fundamental characteristics of wave scattering in attenuation and dispersion are used to be studied. For solving the problem of plane vibration in engineering, most methods are difficult to identify small defects such as cracks. The reason is that small defects are related to high frequency effects. The latest technique for identifying small defects is shown by using the result of elastic wave motion in high frequency propagation and reflection. Stawiarski et al. [10] adopt the elastic wave to detect the initiation of the fatigue damage in isotropic plate. The propagation and reflection of waves indicate a bright path for nondestructive testing of structures. Dubuc et al. [11] used the three-dimensional numerical model to analyze guided wave motion with tensile and shear cracks in isotropic plane. Komijani et al. [12] adopted enhanced finite element models to solve dynamic crack propagation and wave propagation.

Numerical simulation has become the key factor of product design in engineering for the latest decades. To reduce the costs and product development time in engineering, many researchers adopt numerical simulation techniques to guide their design process, which is called conceptual design 
[13]. With the development of commercial software and computer resources, many engineers can use the numerical models to analyze more complex structures for fault diagnosis and for identification at higher frequencies [14]. At present, the most popular numerical modeling technique for high frequency wave motion is finite element method. Manktelow et al. [5] used integrated commercial software which can explore and optimize the complex structure to analyze nonlinear wave dispersion. The finite element method is used to accurately simulate the high frequency wave motion analysis. The method needs at least 20 nodes which are generated by a lot of elements to simulate a wavelength that leads to a huge computational cost. Spatial discretization problem is the main problem that high frequency wave motion faced in engineering. By fine and accurate processing, the space structure, the wave motion dispersion, and response of the structure can be obtained. Nanda et al. [15] present a spectral finite element method which can adopt the efficient and accurate layerwise theory to analyze the nonuniform composite and sandwich structure beams. However, spectral finite element methods require modifying two-dimensional or three-dimensional problems, geometrical complexities, nonperiodic boundary conditions, and so on. Guo et al. [16] solved linear wave motion equations by adopting a fully discrete element method.

Due to the uncertainty of modeling, even a small error can cause a large error of high frequency fluctuation. Therefore, the accuracy of structural models is very important. Park et al. [17] proposed a generalized multiscale finite element method to simulate the question of fluid flows. This paper considered a coupled factor of two equations, which can fine grid in order to improve accuracy. Wavelets have been widely used in many physics and engineering problems in recent years [18]. Due to the fact that the wavelet has the characteristic of multiresolution analysis, it provides a new mechanism that can decompose the solution into a series of coefficients. The numerical method of wavelet functions can be regarded as finite element shape functions. It seems like the shape functions of signal or image processing. Basu et al. [19] pointed out that the finite element, the boundary element, and the meshless methods have already replaced the finite difference methods and Ritz methods. Those methods may be replaced by the wavelet numerical methods in the near future. Chen and Ma [20, 21] constructed beam and plane Daubechies wavelet finite element methods. Those can solve the Euler beam and thin plate bending problem. Because B-spline wavelet functions have the characteristics of display expression and high precision and efficiency, many scholars research the finite element method which adopts B-spline wavelet functions as shape functions [22, 23]. Further, Xiang et al. [24] proposed Hermite cubic spline wavelet to solve intensity factors. Xiang indicated that the Hermitian scale and wavelets functions should be truncated. Xue et al. [25] presented a modified Hermitian interpolation wavelet base by adding the appropriate wavelet function to solve the wave motion and load identification. However, these interpolation functions satisfy the elements of $C_{0}$, whose functions can be interpolated for the displacement. The method of interpolating the rotation by calculating the first derivative of displacement makes the accuracy of Hermitian spline wavelets be limited. Moreover, these interpolation functions are very complex, which contain more nodes in a wavelet element. Although the accuracy of the wavelet is very high, the amount of calculation is very large.

The new effective wavelet finite element methods that adopt Hermitian wavelet functions as shape functions are presented in this paper. The new Hermitian wavelet plane element is called Hermitian spline wavelet on interval (HSWI) element. The new Hermitian wavelet functions satisfy the elements of $C_{1}$, whose functions can be interpolated for the displacement and rotation at the same time. Moreover, this new plane element has a very small number of nodes. The accuracy is higher than that of the Hermitian element constructed by the authors in [26]. The wavelet functions are orthogonal under the condition of given inner product. These new shape functions can decouple totally or partially for the Hermitian plane wavelet element. The precision of element can rise by improving the scale and nesting the approximation space.

\section{Hermitian Plane Wavelet Finite Element}

2.1. Hermitian Plane Wavelet Shape Functions. The scale functions $\phi_{1, k}$ and wavelet functions $\psi_{1, k}$ of Hermitian wavelet are shown in Figure 1.

The equations of scale functions $\phi_{1, k}$ are

$$
\left\{\begin{array}{l}
\phi_{1,1}(x)=\sqrt{\frac{5}{24}} \phi_{1}(2 x-1), \\
\phi_{1,2}(x)=\sqrt{\frac{15}{4}} \phi_{2}(2 x), \\
\phi_{1,3}(x)=\sqrt{\frac{15}{8}} \phi_{2}(2 x-1), \\
\phi_{1,4}(x)=\sqrt{\frac{15}{4}} \phi_{2}(2 x-2) .
\end{array}\right.
$$

The wavelet functions $\phi_{1, k}$ of Hermitian are

$$
\left\{\begin{array}{l}
\psi_{j, k}(x)=\frac{2^{-j / 2}}{\sqrt{729.6}} \psi_{1}\left(2^{j} x-\frac{k}{2}\right), \quad \text { when } k \text { is even, } \\
\psi_{j, k}(x)=\frac{2^{-j / 2}}{\sqrt{153.6}} \psi_{2}\left(2^{j} x-\frac{k-1}{2}\right), \quad \text { when } k \text { is odd }, \\
\psi_{j, 1}(x)=\frac{2^{-j / 2}}{\sqrt{76.8}} \psi_{2}\left(2^{j} x\right) \\
\psi_{j, 2^{j+1}}(x)=\frac{2^{-j / 2}}{\sqrt{76.8}} \psi_{2}\left(2^{j} x-2^{j}\right)
\end{array}\right.
$$




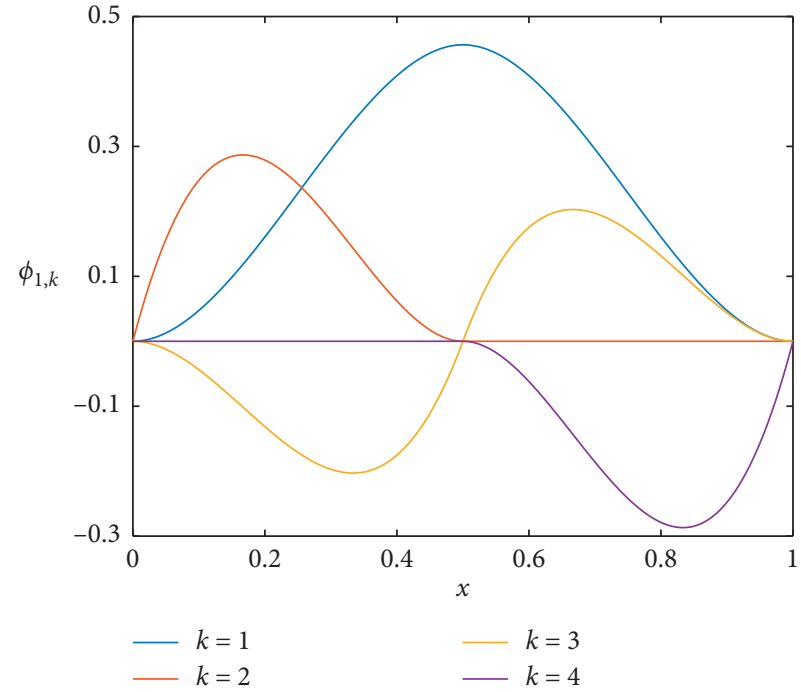

(a)

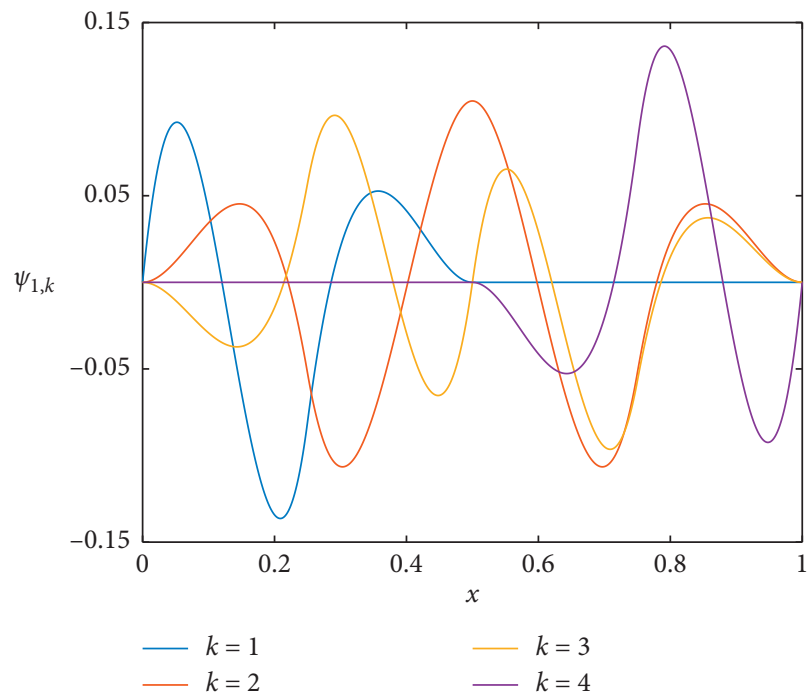

(b)

Figure 1: Hermitian scale and wavelet functions. (a) Scale functions. (b) Wavelet functions (scale $j=1$ ).

where

$$
\begin{gathered}
\left\{\begin{array}{l}
\psi_{1}(x)=-2 \phi_{1}(2 x+1)+4 \phi_{1}(2 x)-2 \phi_{1}(2 x-1)-21 \phi_{2}(2 x+1)+21 \phi_{2}(2 x-1), \\
\psi_{2}(x)=\phi_{1}(2 x+1)-\phi_{1}(2 x-1)+9 \phi_{2}(2 x+1)+12 \phi_{2}(2 x)+9 \phi_{2}(2 x-1),
\end{array}\right. \\
\phi_{1}(x)= \begin{cases}(x+1)^{2}(1-2 x), & -1 \leq x \leq 0, \\
(1-x)^{2}(1+2 x), & 0 \leq x \leq 1, \\
0, & \text { other, }\end{cases} \\
\phi_{2}(x)= \begin{cases}(x+1)^{2} x, & -1 \leq x \leq 0, \\
(x-1)^{2} x, & 0 \leq x \leq 1, \\
0, & \text { other. }\end{cases}
\end{gathered}
$$

The shape functions of finite element method should satisfy the necessary condition that the sum of shape functions at any node is 1; the original scale functions of Hermitian wavelet cannot satisfy the conditions at the boundary node, which is called boundary problem. Stretching and translating can make some of Hermitian functions meet the requirements of Lagrange interpolation and the other meet the requirements of Hermite interpolation functions.

The new Hermitian interpolation functions are constructed by the Hermitian scale and wavelet functions. The equations are

$$
\begin{cases}\phi_{j, k}(x)=\phi_{1}\left(2^{j} x-\frac{k-1}{2}\right), & \text { when } k \text { is odd, } \\ \phi_{j, k}(x)=\frac{1}{2^{j}} \phi_{2}\left(2^{j} x-\frac{k-2}{2}\right), & \text { when } k \text { is even. }\end{cases}
$$

These functions can be interpolated for the displacement when $k=1,3,5, \ldots, 2^{j+1}+1$, as well as for rotation when $k=2,4,6, \ldots, 2^{j+1}+2$. When $k$ is odd number, the interpolation functions satisfy the condition of element $C_{0}$, and the displacement can be interpolated. When $k$ is even number, the interpolation functions satisfy the $C_{1}$ type condition and can interpolate the rotation. Figure 2 is the graph of modified Hermitian wavelet shape functions.

The wavelet space $H_{0}^{1}(0,1)$ can be generated by the scale and wavelet functions; the equations of decomposition are

$$
H_{0}^{1}(0,1)=V_{1} \dot{+} W_{1} \dot{+} W_{2} \dot{+} \cdots,
$$

where $\dot{+}$ is the symbol of direct sum, $V_{1}$ represents the initial scale space, the wavelet space is $W_{j}$, and $j$ is different level of wavelet.

The equations of Hermitian wavelet shape function are

$$
\Phi=\left[\begin{array}{llll}
\phi_{1}^{j} & \phi_{2}^{j} & \cdots & \phi_{2^{j+1}+2}^{j}
\end{array}\right] .
$$




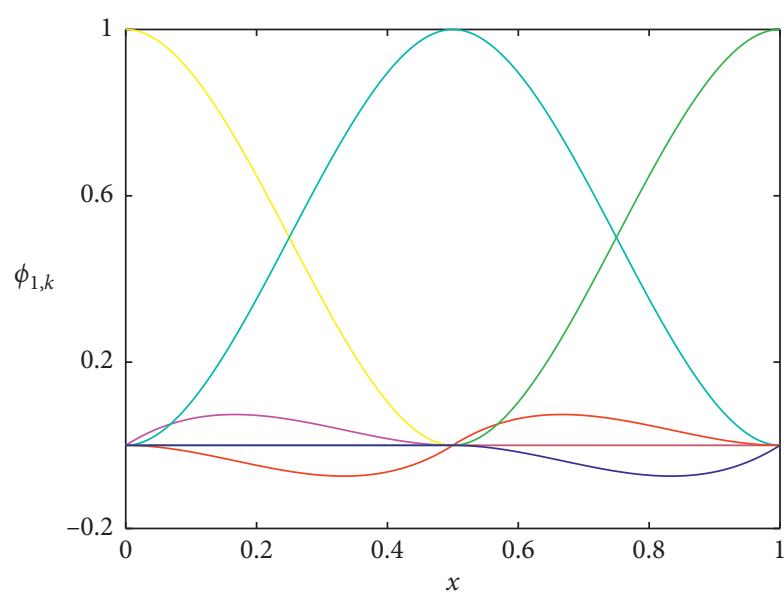

(a)

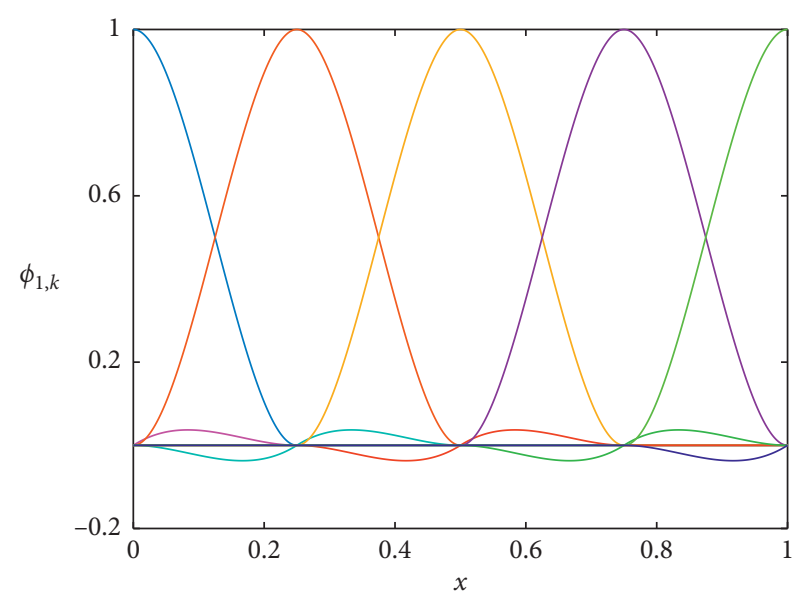

(b)

FIgURE 2: Hermitian wavelet shape functions. (a) Scale $j=1$; (b) scale $j=2$.

The Hermitian wavelet functions have an excellent characteristic: the first derivative has good continuity, which makes modified Hermitian wavelet functions meet interpolation condition of $C_{1}$. These features are more suitable for the research of beam, plane, and so on. With finite element shape functions, HSWI can solve the engineering problems with high precision.

Figure 2 shows the new scale functions $\phi_{j, k}$, the corresponding approximation space is $H_{0}^{1}(0,1)$, and the intervals of HSWI shape functions are $[0,1]$. Using the tensor product, the Hermitian plane wavelet approximation space $H_{0}^{1}(0,1)$ is constructed. The initial scale space is $V_{1}$, the wavelet space is $W_{j}$, and $j$ is different level of wavelet. So the subspace which generates tensor product is $\varphi_{j}=\Phi_{j} \otimes \Phi_{j}$, the scale functions are $\varphi_{1}=\Phi_{1} \otimes \Phi_{1}$ at $j=1$, the scale functions are $\varphi_{2}=\Phi_{2} \otimes \Phi_{2}$ at $j=2$, and so on. Figure 3 shows the tensor product of HSWI plane elements where the scale is $j=1,2$.

The plane Hermitian wavelet shape functions can replace the traditional finite element shape functions. Using the new shape functions, stiffness matrix and mass matrix can be solved. The Newmark time integration can calculate the results of high frequency wave motion by the stiffness matrix and mass matrix.

2.2. Hermitian Wavelet Finite Element Formula. The elements are divided into two types in plane structure: one is plane stress element, and the other is plane strain element. Plane stress element is established based on the Hermitian wavelet shape functions. The plane strain element can use $E /\left(1-\mu^{2}\right)$ and $\mu /(1-\mu)$ to replace $E$ and $\mu$, where $E$ is Young's modulus, and $\mu$ is Poisson's ratio.

For the plane structure, the formula of potential energy is

$$
\Pi(u)=\int_{\Omega}{ }_{-} \varepsilon^{T} J \varepsilon h \mathrm{~d} x \mathrm{~d} y-\int_{\Omega} u^{T} f h \mathrm{~d} x \mathrm{~d} y-\int_{S_{\sigma}} u^{T} p h \mathrm{~d} s,
$$

where $h$ represents the thickness of element, $f=\left\{f_{x}, f_{y}\right\}$ shows the body forces vector, the displacements vector is $u=\{u, v\}^{T}$, and the surface traction vector is $P=\left\{p_{x}, p_{y}\right\}^{T}$, where $p_{x}$ represents $x$ directions and $p_{y}$ represents $y$ directions. $J$ is the elastic matrix; $\varepsilon=\left\{\varepsilon_{x}, \varepsilon_{y}, \gamma_{x y}\right\}^{T}$ is strain matrix:

$$
\begin{aligned}
& J=\frac{E}{1-\mu^{2}}\left[\begin{array}{ccc}
1 & \mu & 0 \\
\mu & 1 & 0 \\
0 & 0 & \frac{1-\mu}{2}
\end{array}\right], \\
& \varepsilon=\left[\begin{array}{cc}
\frac{\partial}{\partial x} & 0 \\
0 & \frac{\partial}{\partial y} \\
\frac{\partial}{\partial y} & \frac{\partial}{\partial x}
\end{array}\right] u .
\end{aligned}
$$

Assume that the material has the characteristic of being linearly elastic and isotropic; the stress equation is

$$
\sigma=\left\{\begin{array}{c}
\sigma_{x} \\
\sigma_{y} \\
\tau_{x y}
\end{array}\right\}=J \mathcal{\varepsilon} .
$$

Adopting the scale $j=2$ as an example, the arrangement of plane nodes is shown in Figure 4; the displacement functions in $x$ direction and $y$ direction are

$$
\begin{aligned}
& u(\xi, \eta)=\Phi T u \\
& v(\xi, \eta)=\Phi T v .
\end{aligned}
$$

$\Phi$ represents the plane Hermitian wavelet shape functions, $T$ shows the transformation matrix which is from wavelet space to physical space, the displacement in $x$ direction is $u$, and in $y$ direction it is $v$ : 


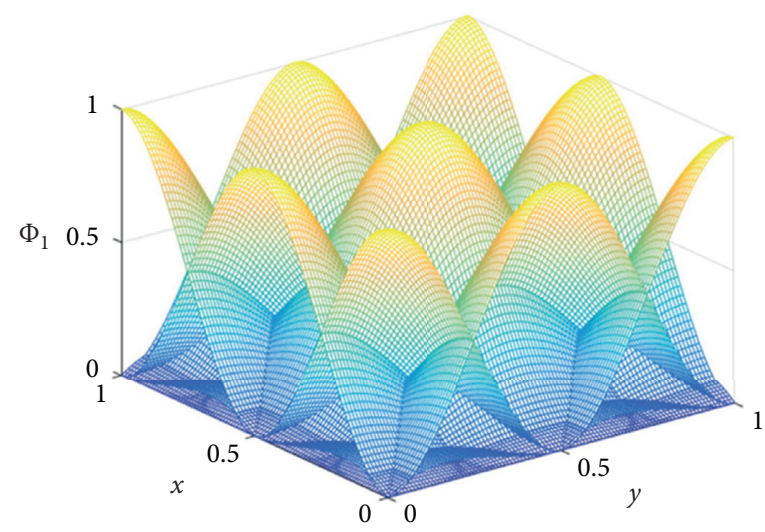

(a)

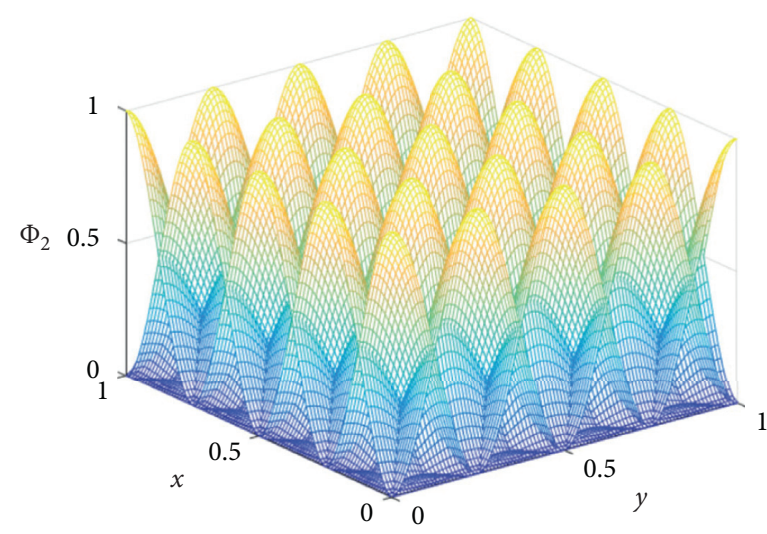

(b)

FIgURE 3: Tensor product of Hermitian wavelet shape functions. (a) Scale $j=1$; (b) scale $j=2$.

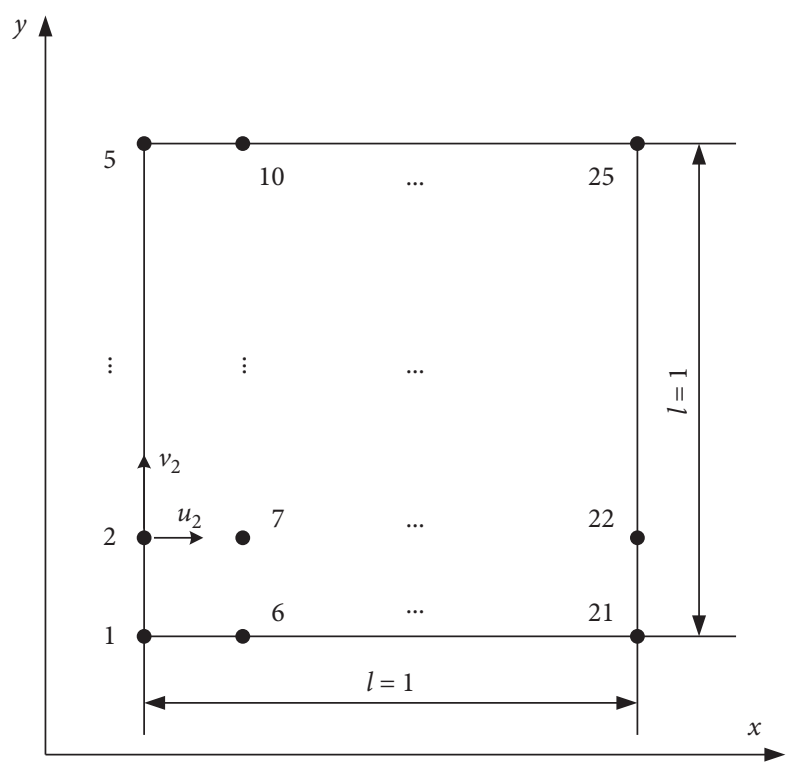

FIGURE 4: Arrangement of plane nodes.

$$
\begin{gathered}
u^{e}=\left\{\begin{array}{c}
u_{1} \\
u_{2} \\
\vdots \\
u_{(n+1)^{2}-1} \\
u_{(n+1)^{2}}
\end{array}\right\}, \\
v^{e}=\left\{\begin{array}{c}
v_{1} \\
v_{2} \\
\vdots \\
v_{(n+1)^{2}-1} \\
v_{(n+1)^{2}}
\end{array}\right\} .
\end{gathered}
$$

The standard element domain can be gained by mapping the original element domain. Substituting equations (8) and (9) into equation (7), the principle of Galerkin variation can obtain the finite element equations:

$$
\left[\begin{array}{ll}
K_{1}^{e} & K_{2}^{e} \\
K_{3}^{e} & K_{4}^{e}
\end{array}\right]\left[\begin{array}{l}
u^{e} \\
v^{e}
\end{array}\right]=\left[\begin{array}{c}
\left(T^{e}\right)^{T} \int_{S_{\sigma}} p_{x} \Phi^{T} \mathrm{~d} s+\int_{\Omega} f_{x} \Phi^{T} \mathrm{~d} \Omega \\
\left(T^{e}\right)^{T} \int_{S_{\sigma}} p_{y} \Phi^{T} \mathrm{~d} s+\int_{\Omega} f_{y} \Phi^{T} \mathrm{~d} \Omega
\end{array}\right],
$$

where

$$
\begin{aligned}
& K_{1}^{e}=\frac{E}{\left(1-\mu^{2}\right)}\left(\frac{G_{1}^{11} \otimes G_{2}^{00}+(1-\mu)}{2 G_{1}^{00} \otimes G_{2}^{11}}\right), \\
& K_{4}^{e}=\frac{E}{\left(1-\mu^{2}\right)}\left(\frac{G_{1}^{00} \otimes G_{2}^{11}+(1-\mu)}{2 G_{1}^{11} \otimes G_{2}^{00}}\right), \\
& K_{2}^{e}=\frac{E}{\left(1-\mu^{2}\right)}\left(\frac{\mu G_{1}^{10} \otimes G_{2}^{01}+(1-\mu)}{2 G_{1}^{01} \otimes G_{2}^{10}}\right), \\
& K_{3}^{e}=\left(K_{2}^{e}\right)^{T} .
\end{aligned}
$$

The equation of consistent mass matrix is

$$
M^{e}=\rho A h\left(T^{e}\right)^{T}\left(\frac{\partial\left(G_{1}^{00} \otimes G_{2}^{00}\right)^{T}}{\partial \xi}\right)\left(\frac{\partial\left(G_{1}^{00} \otimes G_{2}^{00}\right)}{\partial \xi}\right) T^{e},
$$

where the density is $\rho$, the plane area is $A, G_{1}^{00}=l_{\text {ex }}$ $\int_{0}^{1} \Phi^{T} \Phi \mathrm{d} \xi, G_{1}^{01}=\int_{0}^{1} \Phi\left(\mathrm{d} \Phi^{T} / \mathrm{d} \xi\right) \mathrm{d} \xi, G_{1}^{10}=l_{e x} \int_{0}^{1}\left(\mathrm{~d} \Phi^{T} / \mathrm{d} \xi\right) \Phi$ $\mathrm{d} \xi$, and $G_{1}^{11}=1 / l_{e x} \int_{0}^{1}\left(\mathrm{~d} \Phi^{T} / \mathrm{d} \xi\right)(\mathrm{d} \Phi / \mathrm{d} \xi) \mathrm{d} \xi$.

Similarly, $G_{2}^{i j}(i, j=1,2)$ can be easily obtained. $l_{e y}$ and $\mathrm{d} \eta$ can replace $l_{e x}$ and $\mathrm{d} \xi$.

The plane Hermitian wavelet shape functions are constructed by using the tensor product of modified Hermitian wavelet element in this paper. Substituting the plane Hermitian wavelet shape functions into the finite element formula, the stiffness matrix and mass matrix can be gained.

The stiffness matrix can be converted from the wavelet domain to the physical domain. Similarly, the 


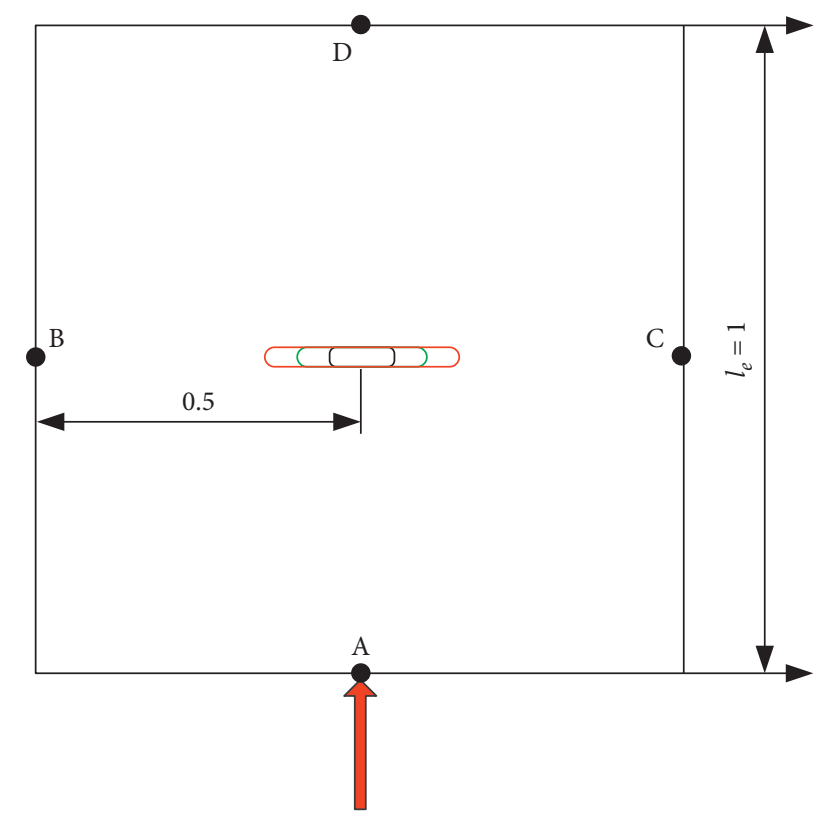

FIgURE 5: Arrangement for cracks in plane structure.

degrees of freedom of element can be transformed from the wavelet coefficients to physical field functions. So, the continuity and compatibility between adjacent elements can be satisfied. Because $u$ and $v$ do not have coupled factors, they are independent displacements. The transformation matrix of plane can be constructed by solving the tensor product of modified shape functions in this paper. The other steps are like the traditional finite element method.

The wave motion rule is solved by the motion equation of finite element method. The finite element method can be discretized in space domain and the motion equation can be dispersed in time domain. The Newmark time integration method can be applied:

$$
M \ddot{u}(t)+C \dot{u}(t)+K u(t)=F(t),
$$

where $K$ is the stiffness matrix, the mass matrix is $M, C$ represents the damping matrix, the excitation force is $F(t)$, and $u, \dot{u}$, and $\ddot{u}$ are displacement, velocity, and acceleration, respectively. The Rayleigh damping formula is used to calculate the damping matrix in this paper.

The velocity and displacement which are in Newmark time integration are assumed as follows:

$$
\begin{aligned}
\dot{u}^{p+1}(t)= & \dot{u}^{p}(t)+(1-\alpha) \ddot{u}^{p}(t) \Delta t+\alpha \ddot{u}^{p+1}(t) \Delta t, \\
u^{p+1}(t)= & u^{p}(t)+\dot{u}^{p}(t) \Delta t+\left(\frac{1}{2}-\beta\right) \ddot{u}^{p}(t) \Delta t^{2} \\
& +\beta \ddot{u}^{p+1}(t) \Delta t^{2},
\end{aligned}
$$

where $p$ represents the number of time steps and the time interval is $\Delta t$, which is from $p-1$ to $p$ step. Substituting equations (17) and (18) into equation (16), the response of wave motion equation is

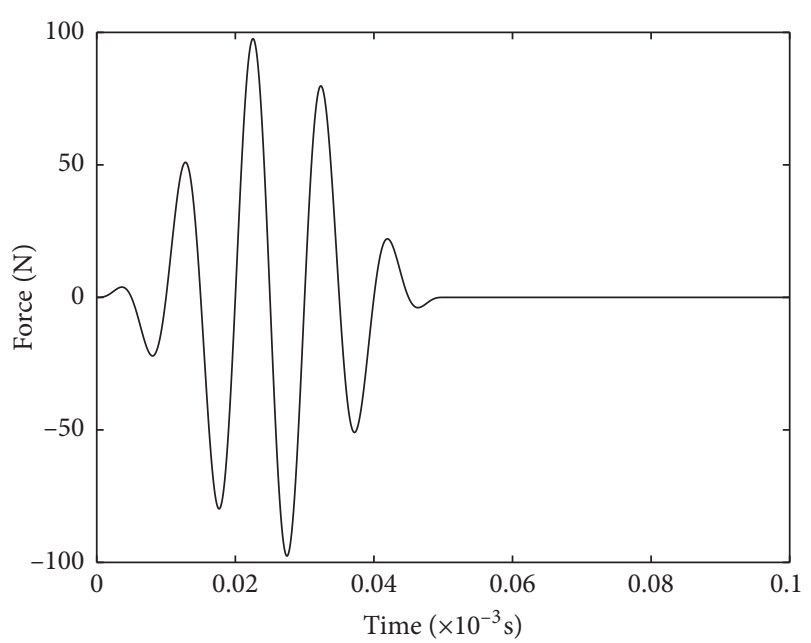

(a)

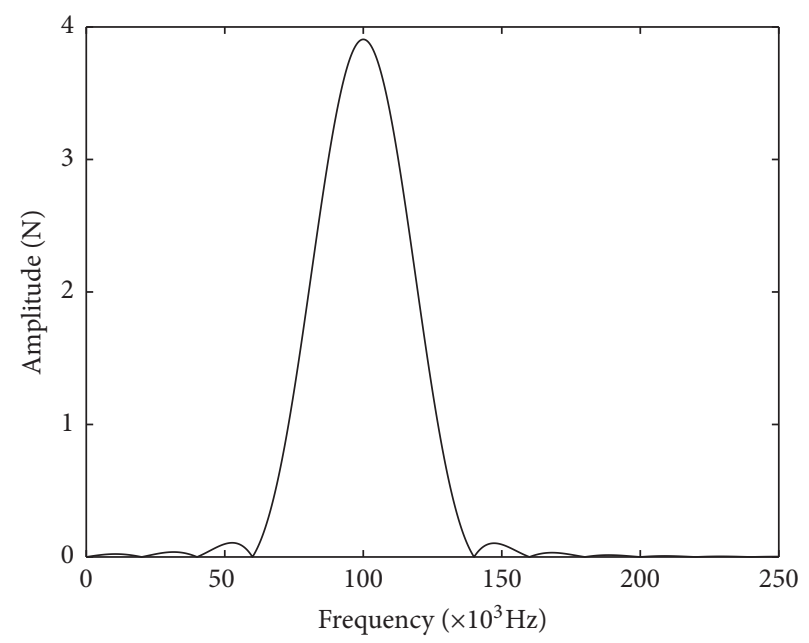

(b)

FIgURE 6: Excitation signal for numerical simulation. (a) Time domain; (b) frequency domain.

$$
\begin{aligned}
u^{p+1}(t)= & \left(K+\frac{1}{\beta \Delta t^{2}} M+\frac{\alpha}{\beta \Delta t} C\right)^{-1} \\
& \left\{F\left(t_{p}\right)+M\left[\frac{1}{\beta \Delta t^{2}} u^{p}(t)+\frac{1}{\beta \Delta t} \dot{u}^{p}(t)\right.\right. \\
& \left.+\left(\frac{1}{2 \beta}-1\right) \ddot{u}^{p}(t)\right]+C\left[\frac{\alpha}{\beta \Delta t} u^{p}(t)+\left(\frac{\alpha}{\beta}-1\right) \dot{u}^{p}(t)\right. \\
& \left.\left.+\left(\frac{\alpha}{2 \beta}-1\right) \Delta t \ddot{u}^{p}(t)\right]\right\} .
\end{aligned}
$$

The wave motion in plane can be analyzed by equation (19). It is assumed that the material is homogenous and isotropic, and the displacements and velocities which are in the initial situation are zero. 

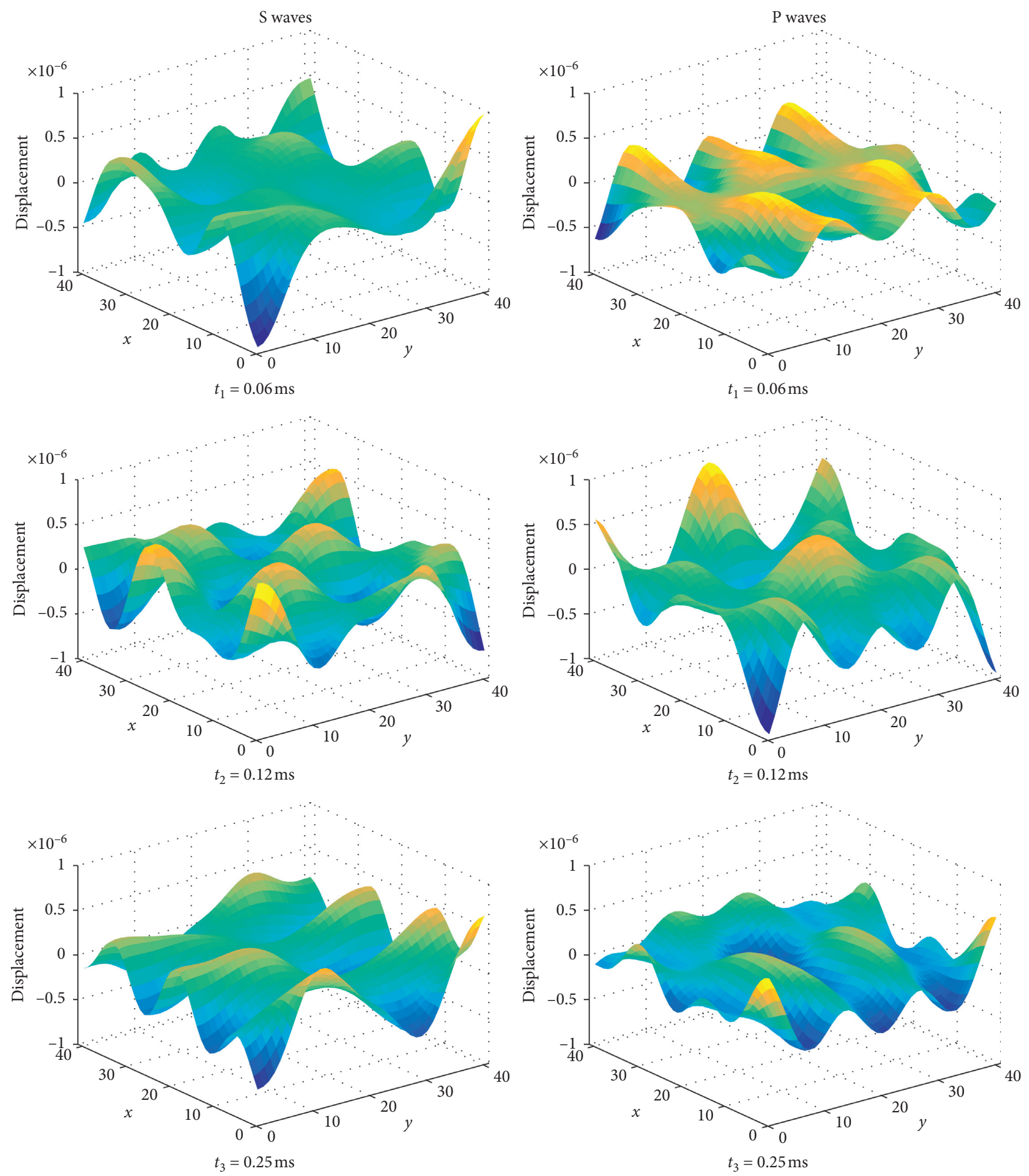

Figure 7: Response results of wave motion without a crack.

\section{Numerical Examples}

The vibration structure especially for the high frequency vibration plays an important role in engineering. The high frequency vibration is also called wave motion. The effect of wave motion is becoming more and more important, and it has significant advantage in detecting small defects, especially cracks. Four working conditions are used to describe plane wave motion analysis in this paper. A sinusoidal signal has a frequency of $100 \mathrm{kHz}$, and the signal is added by Hanning window. The signal is used in this section as the excitation. Figure 5 shows that the excitation location is at point A. Time domain and frequency domain are shown in Figure 6, respectively. The HSWI finite element method is used to analyze wave motion of plane structure in this paper. The wave motion is assumed to be carried out under undamped conditions. 

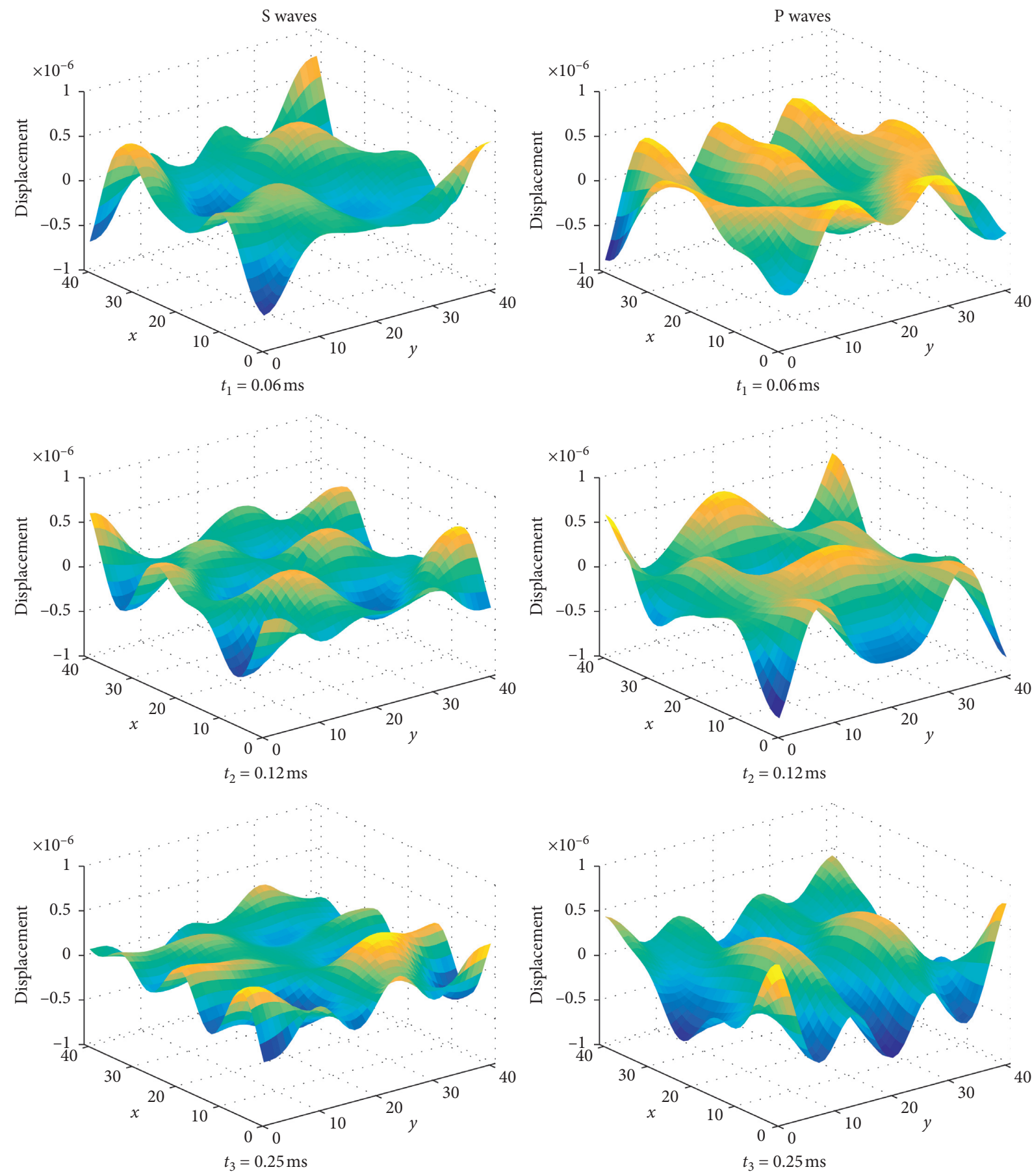

FIGURE 8: Response results of wave motion with crack 1.

The research object is a thin plate of plane, where length and width are $1 \mathrm{~m}$ and the thickness is $0.001 \mathrm{~m}$. Aluminum is used for research in this paper; the material parameters are as follows: Young's modulus is $70 \mathrm{GPa}$, Poisson's ratio is 0.3 , and the density is $2730 \mathrm{~kg} / \mathrm{m}^{3}$. The computer used to calculate the wave motion was composed of Intel CPU, $1.7 \mathrm{GHz}$, and 35G memory. The Matlab software is used under the condition of Windows 10 operating system. Various small defects can be accurately detected by suitable high frequency excitation works on the mechanical structure.
3.1. Analysis of Different Crack Lengths. The assumed small defects crack position is in the middle of plane, and the defects are shown in the form of cracks. The results of wave motion are observed by the response results of three cracks. The three lengths of cracks are $100 \mathrm{~mm}, 200 \mathrm{~mm}$, and $300 \mathrm{~mm}$. The depth of all cracks is half of the plane depth. All the four edges of plane are free boundary conditions. The pulse excitation signal with amplitude of $100 \mathrm{~N}$ is applied in plane shown in Figure 5. The response points are point A, point $\mathrm{B}$, point $\mathrm{C}$, and point $\mathrm{D}$. The wave motion is divided 

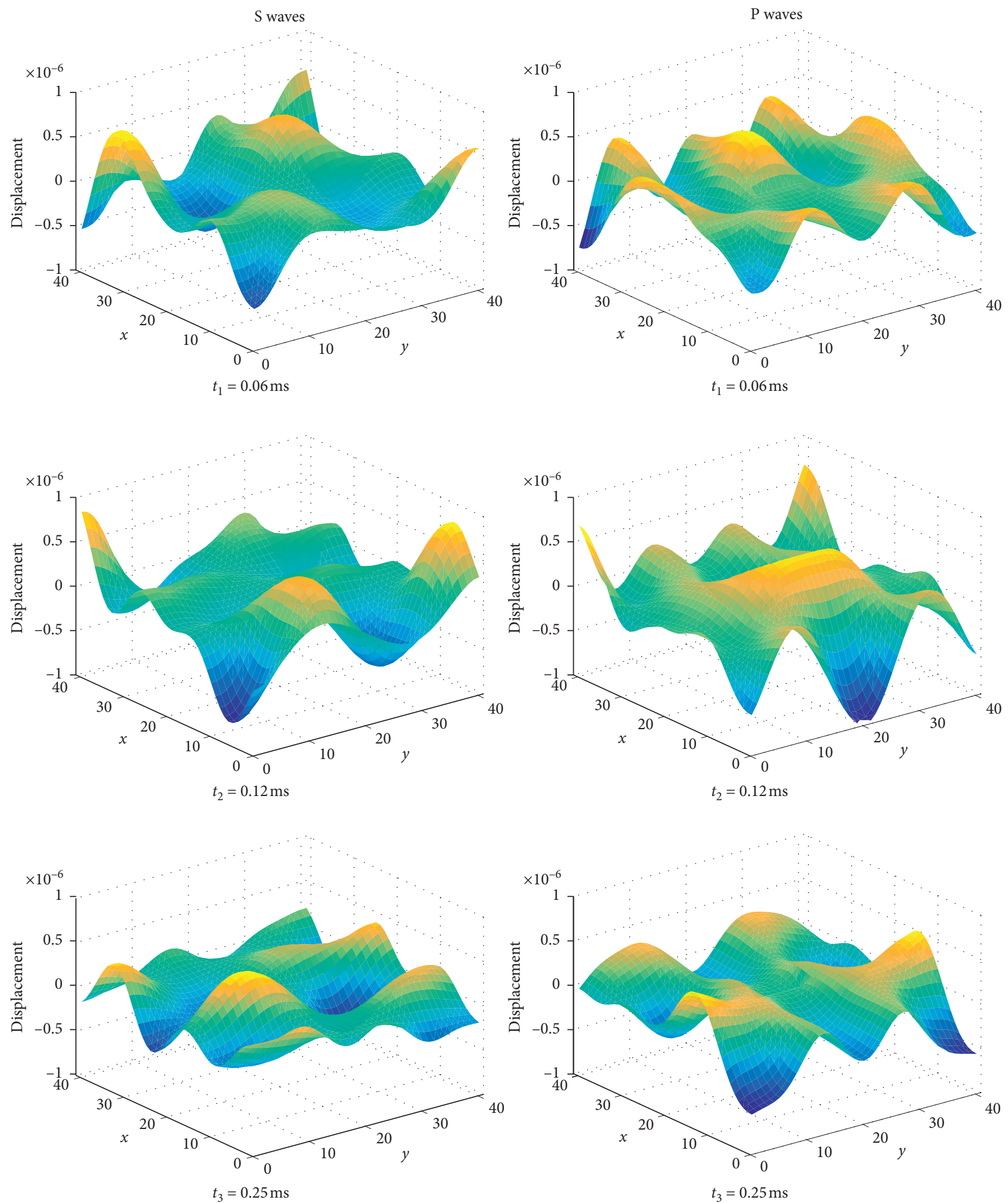

FIGURE 9: Response results of wave motion with crack 2. 

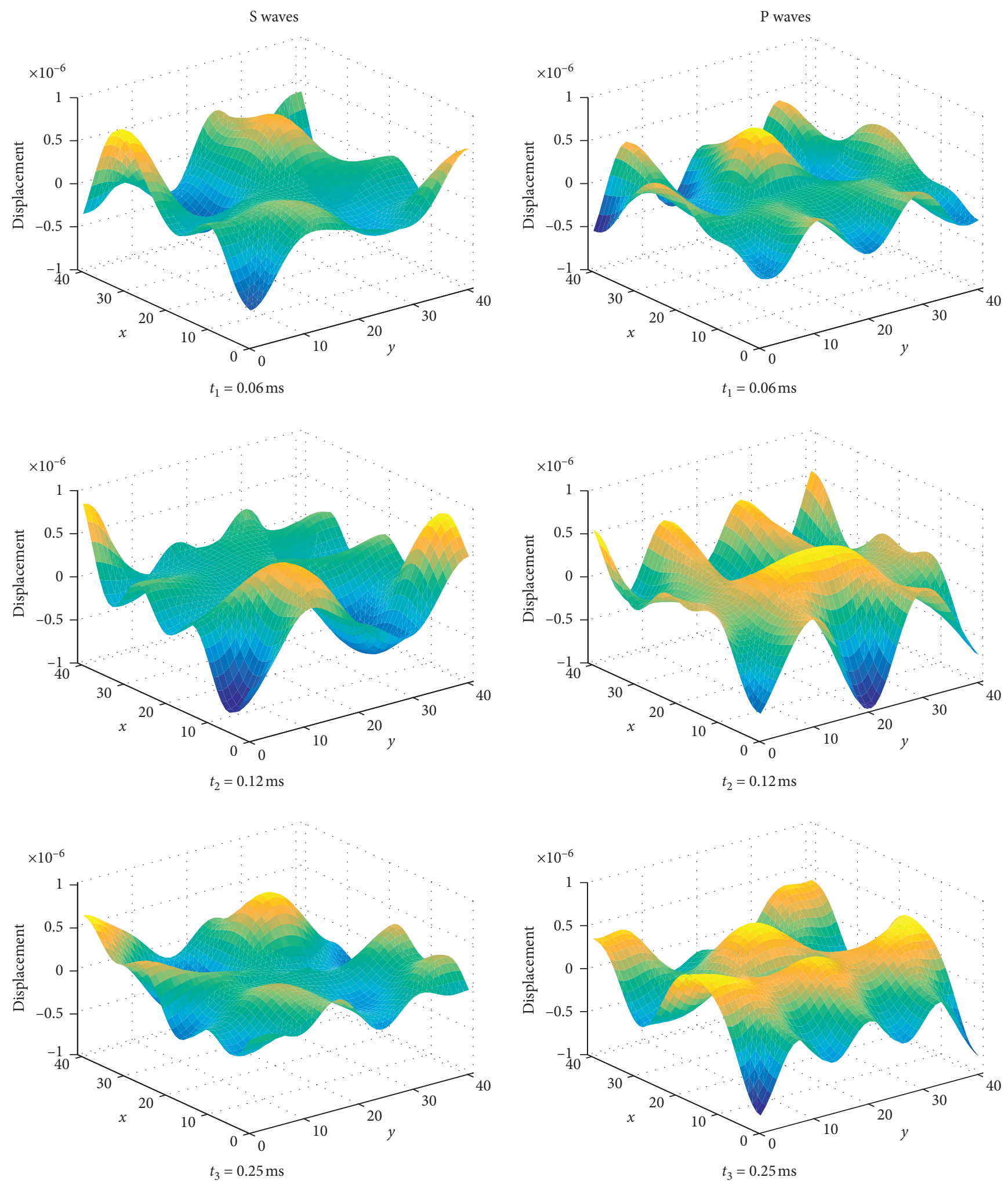

FIGURE 10: Response results of wave motion with crack 3. 

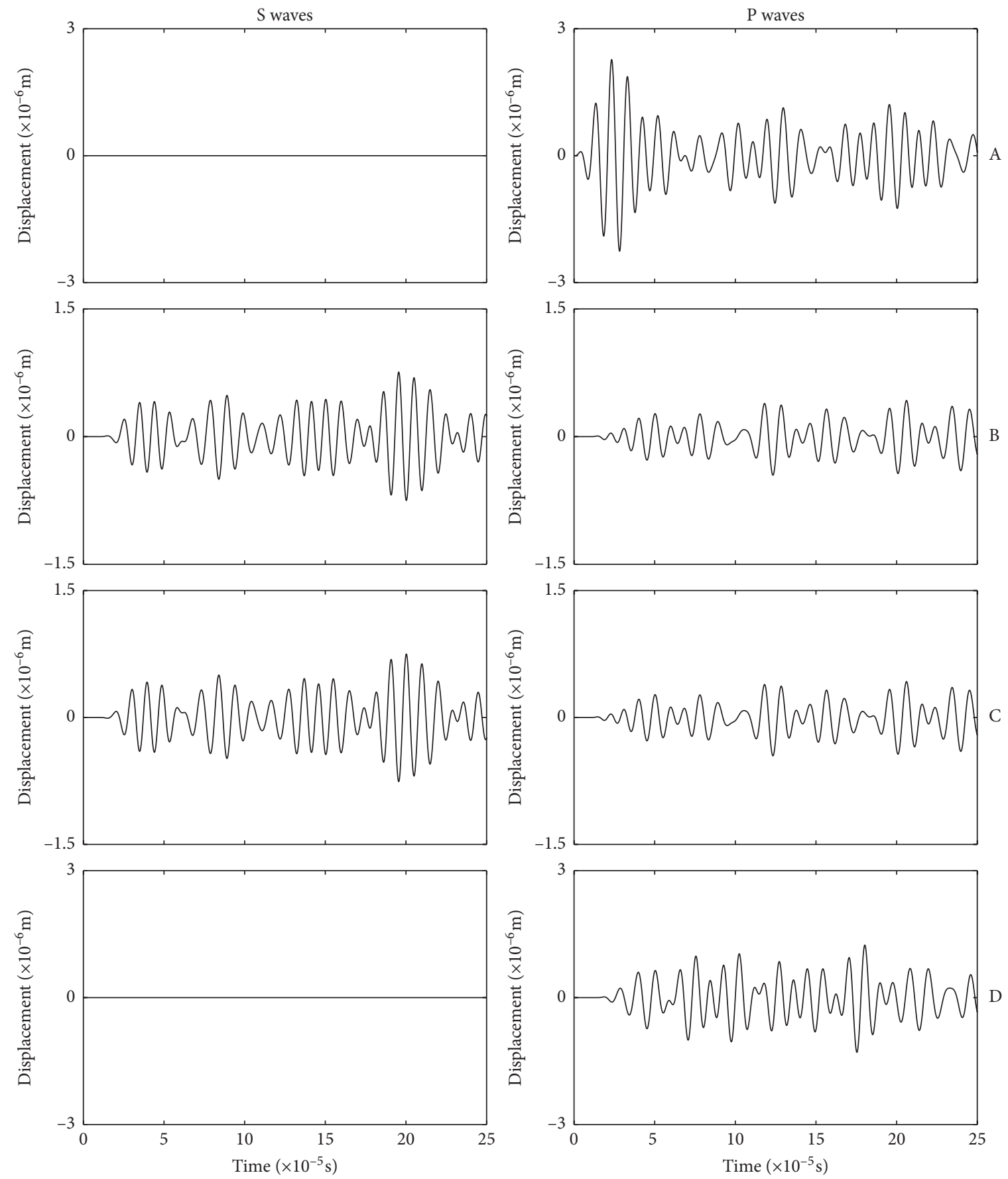

FIgURE 11: Displacement response signals without a crack in the plane.

into the longitudinal wave (or major wave, $\mathrm{P}$ wave) and the transverse wave (or minor wave, $\mathrm{S}$ wave). The influence of wave under the different crack lengths is researched in this section. According to equations (20) and (21), the speed of $\mathrm{P}$ wave and $S$ wave can be solved. The time of wave motion was estimated as $0.25 \mathrm{~ms}$ :

$$
\begin{gathered}
c_{p}=\sqrt{\frac{E}{\left(1-v^{2}\right) \rho}}=5504.5 \mathrm{~m} / \mathrm{s}, \\
c_{s}=\sqrt{\frac{E}{2(1+v) \rho}}=3186 \mathrm{~m} / \mathrm{s} .
\end{gathered}
$$



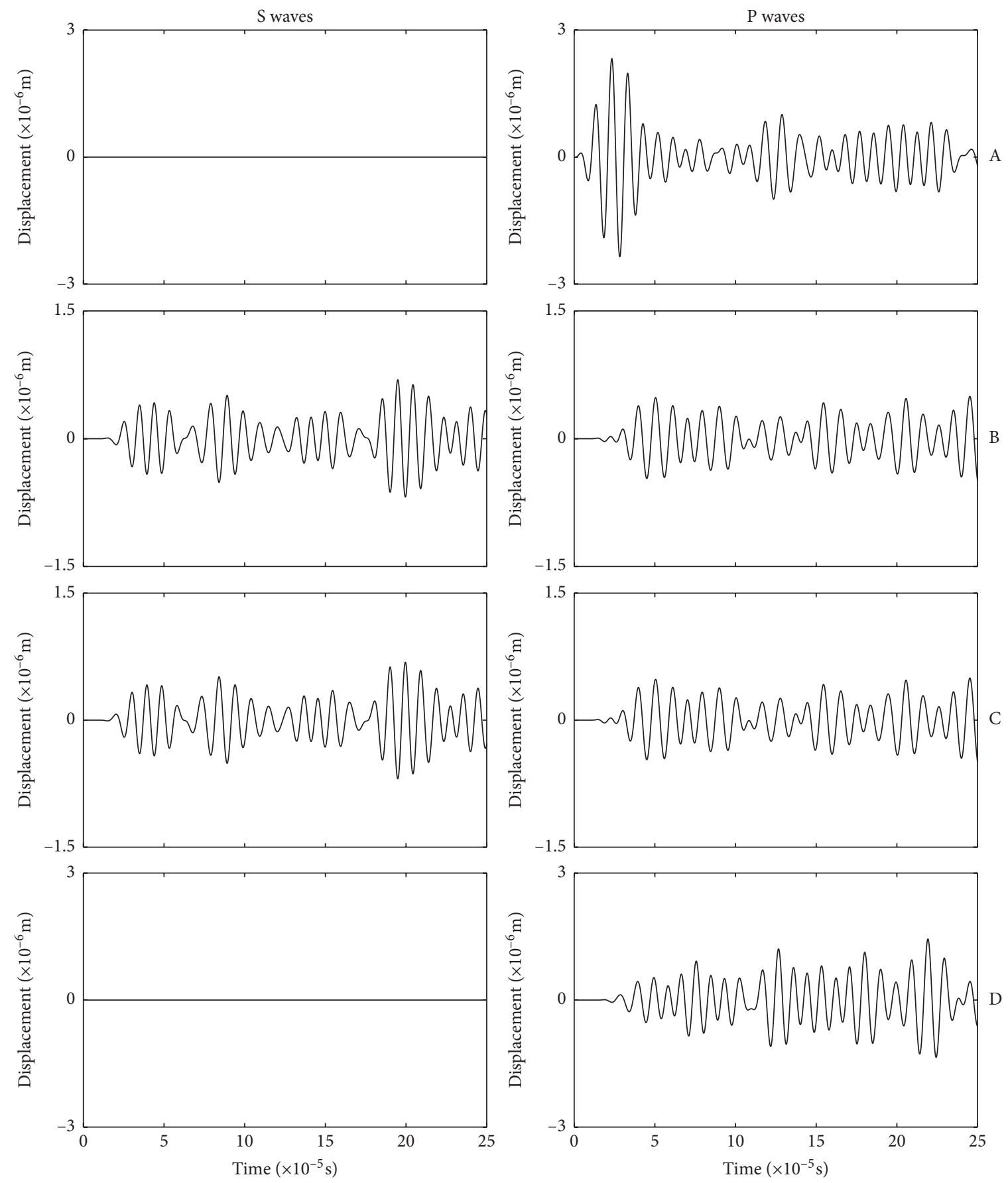

FIGURE 12: Displacement response signals with crack 1 in the plane.

The number of time steps can be divided into 2500 steps in this paper, and the Newmark time integration method is chosen to analyze wave motion. The plane can be divided into $20 \times 20$ regular quadrilateral elements by using the HSWI elements. A HSWI element has 18 degrees of freedom (or 9 nodes), so the plane model has 3,362 degrees of freedom. The $\mathrm{P}$ wave and $\mathrm{S}$ wave are numerically simulated by the HSWI elements with 3362 degrees of freedom in plane. The calculation results are shown in Figures 7-10, where the displacement $v$ refers to $\mathrm{P}$ wave and the displacement $u$ refers to $S$ wave. The graphs of wave motion are displayed at different time.

The difference about wave patterns is obvious with or without crack. The crack is easily identified in the graph of $\mathrm{P}$ 

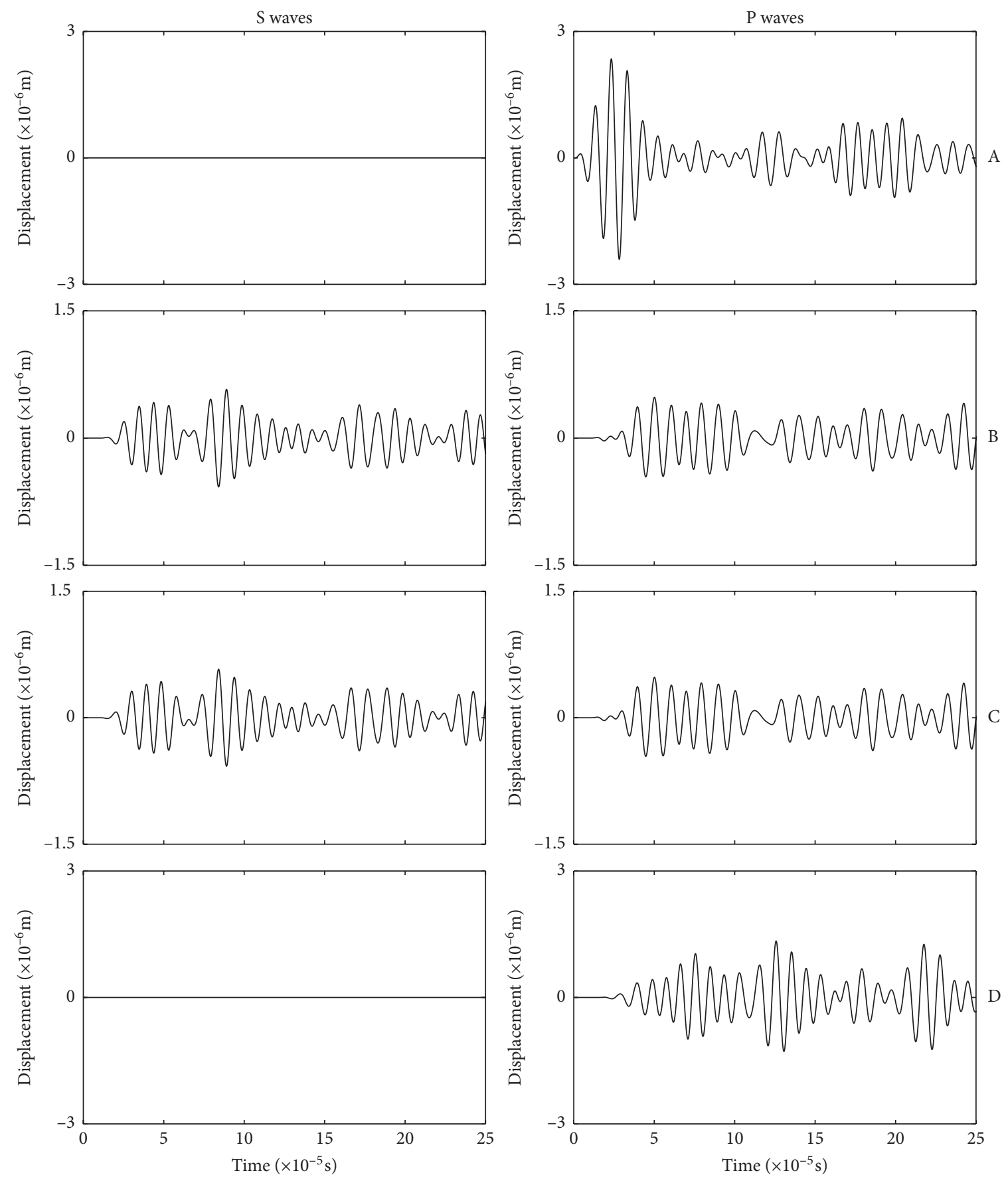

Figure 13: Displacement response signals with crack 2 in the plane.

wave and $S$ wave. The effect of $S$ wave is greater than that of $P$ wave from Figures 7-10. The difference of $\mathrm{P}$ wave is also obvious with or without crack at $0.12 \mathrm{~ms}$ and $0.25 \mathrm{~ms}$. As the length of crack is increased, the waveform of crack becomes more and more obvious. When the length of crack is consistent, the crack waveform becomes larger and larger with time development.
The displacement response diagram at point A, point $\mathrm{B}$, point $C$, and point $D$ is shown in Figures 11-14. The diagram also shows the $\mathrm{P}$ wave and $\mathrm{S}$ wave at each point. The excitation signal is at point $\mathrm{A}$. The displacement response diagram can clearly see whether there is crack or not, as well as the length of crack. As the length of the crack increases, the interval time between the reflected waves and the 

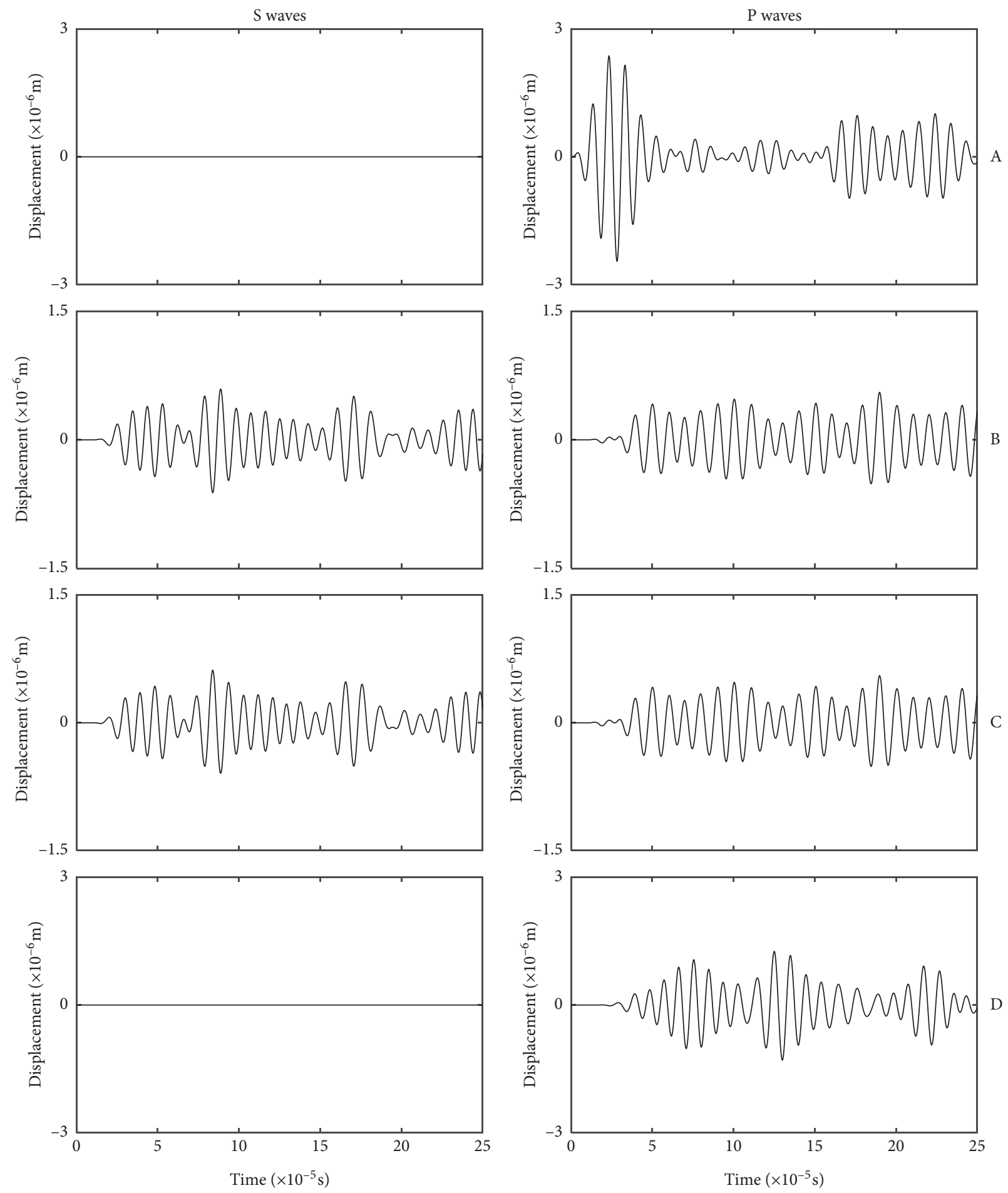

FIGURE 14: Displacement response signals with crack 3 in the plane. 


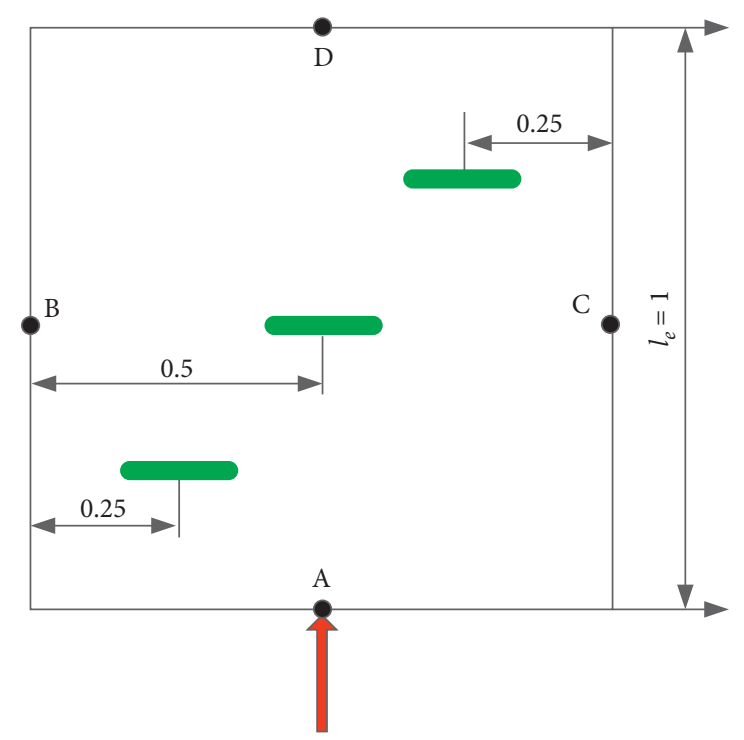

Figure 15: Arrangement for different crack locations.

transmitted waves gets longer and longer at point $\mathrm{A}$. Because the excitation is applied at point $\mathrm{A}$ and the direction is $v$, the response of $\mathrm{S}$ waves does not change in the $u$ direction. Due to the increase of crack length, the response and reflection of $S$ waves are overlapped. Compared without crack situation, the amplitude of $S$ waveform increases significantly, and the modes are denser at point B and C. Because the point D is at the top in the plane, the response of wave is similar to that of point $\mathrm{A}$. The $\mathrm{P}$ waves are greatly affected by the crack at point D. The length of the crack will cause more obvious change of $P$ waves at point $D$.

3.2. Analysis of Different Crack Locations. The cracks are distributed at three locations in plane: the first crack is $0.25 \mathrm{~m}$ at the top and right end, the second crack is in the middle of the plane, and the third crack is $0.25 \mathrm{~m}$ at the bottom and left end. Assume that the length of three cracks is $200 \mathrm{~m}$, and the depth of them is $0.5 \mathrm{~mm}$. The boundary condition is free for four edges in plane. Figure 15 is the plane distributed graph of the crack. HSWI elements are used to analyze high frequency wave motion in plane, and the material is aluminum. An excitation signal in the form of a force pulse signal with amplitude of $100 \mathrm{~N}$ is applied at point $\mathrm{A}$. The response results are observed at point $\mathrm{A}$, point $\mathrm{B}$, point $\mathrm{C}$, and point $\mathrm{D}$ in this section. The wave patterns of $\mathrm{P}$ wave and $\mathrm{S}$ wave are investigated at different crack positions.

The numerical modeling is established in Figure 15 by the use of HSWI elements. Figures 16-19 display the calculation results at different positions for cracks. It is worth mentioning that the waveform and displacement response graphs are Figures 9 and 13 for the second crack. The cracks under different positions are expanded on analysis at different time points.

The propagation and reflection of waves will change greatly when the crack location changes. The crack location 2 is in the middle (Figure 9), and the influence of $\mathrm{P}$ waves is greater than $S$ waves compared with the waveform of without crack (Figure 7). The crack locations 1 and 3 have great influence on $\mathrm{P}$ and $\mathrm{S}$ waves compared with the waveform without crack (Figures 16 and 17). Compared to the transmitted waves, the reflected crack waves are dominant in the $\mathrm{P}$ waves. So the $v$ displacement response is worth researching.

The wave motion graphs of $P$ wave and $S$ wave, where the excitation signal is applied at point $A$, were displayed in Figures 13, 18, and 19. However, the response signals are observed at point $\mathrm{A}$, point $\mathrm{B}$, point $\mathrm{C}$, and point $\mathrm{D}$. The wave pattern varies greatly for the cracks at different positions. When the crack is not in the middle, it can be seen from Figures 18 and 19 that there are additional $S$ waves at point $A$ and point $D$. But there was no $S$ wave at point $A$ and point $D$ under the absence of crack (Figure 11). When the crack is in the middle, there is no additional waveform at point $A$ and point $\mathrm{D}$. The wave propagation and wave reflection have a certain interval time. Due to the influence of crack position, the $S$ wave pattern and amplitude have changed obviously at point $\mathrm{B}$ and point $\mathrm{C}$. This phenomenon can be used to identify the location of different cracks. The cracks will reduce the amplitude of waves at point $\mathrm{D}$ in the $v$ direction displacement. The geometry is similar to literature [3], with $40 \times 40$ spectral elements, and a total of 80,802 degree of freedom are used in this literature. In this paper, the new 

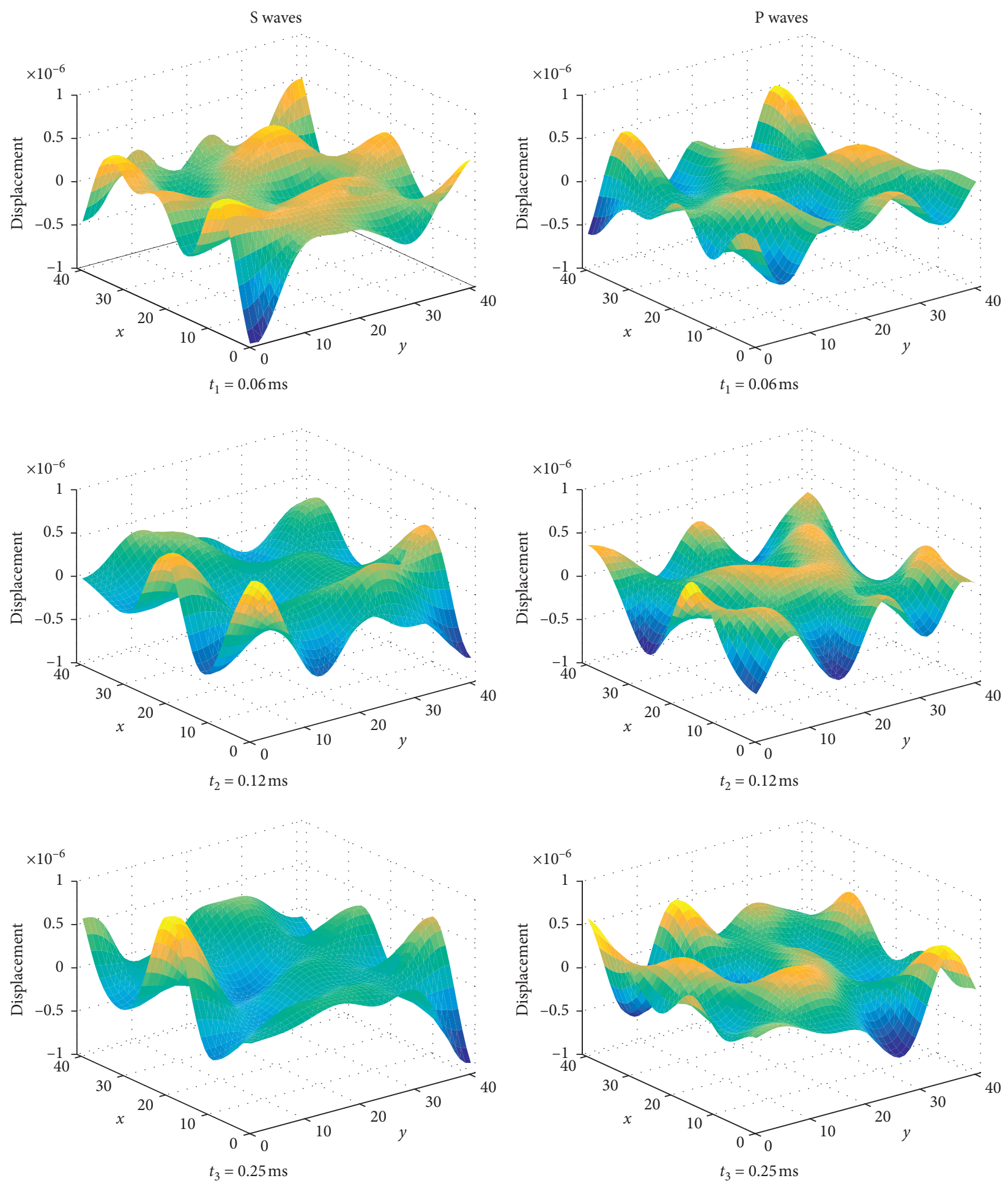

Figure 16: Response results of wave motion with location 1. 

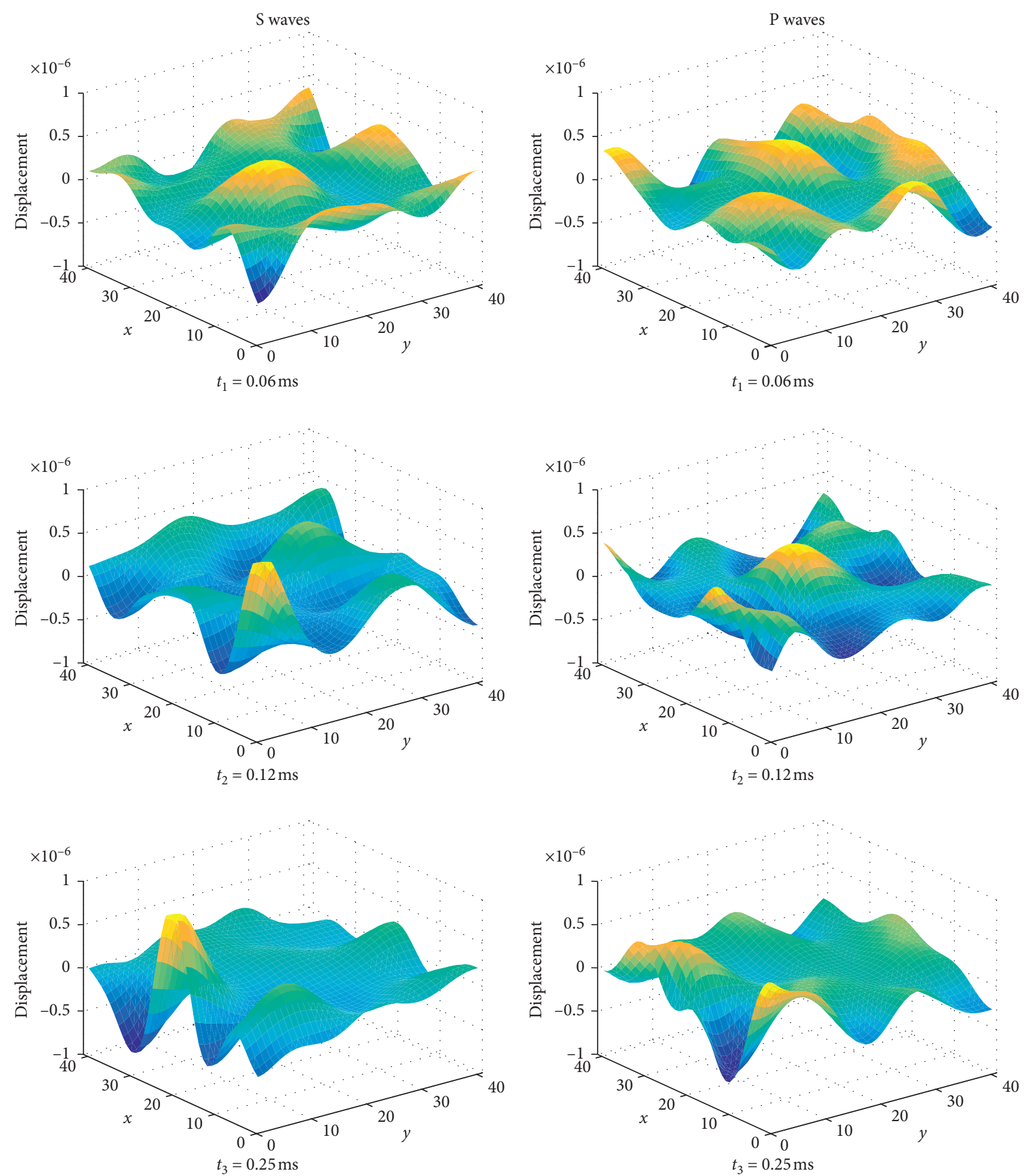

FIgURE 17: Response results of wave motion with location 3. 

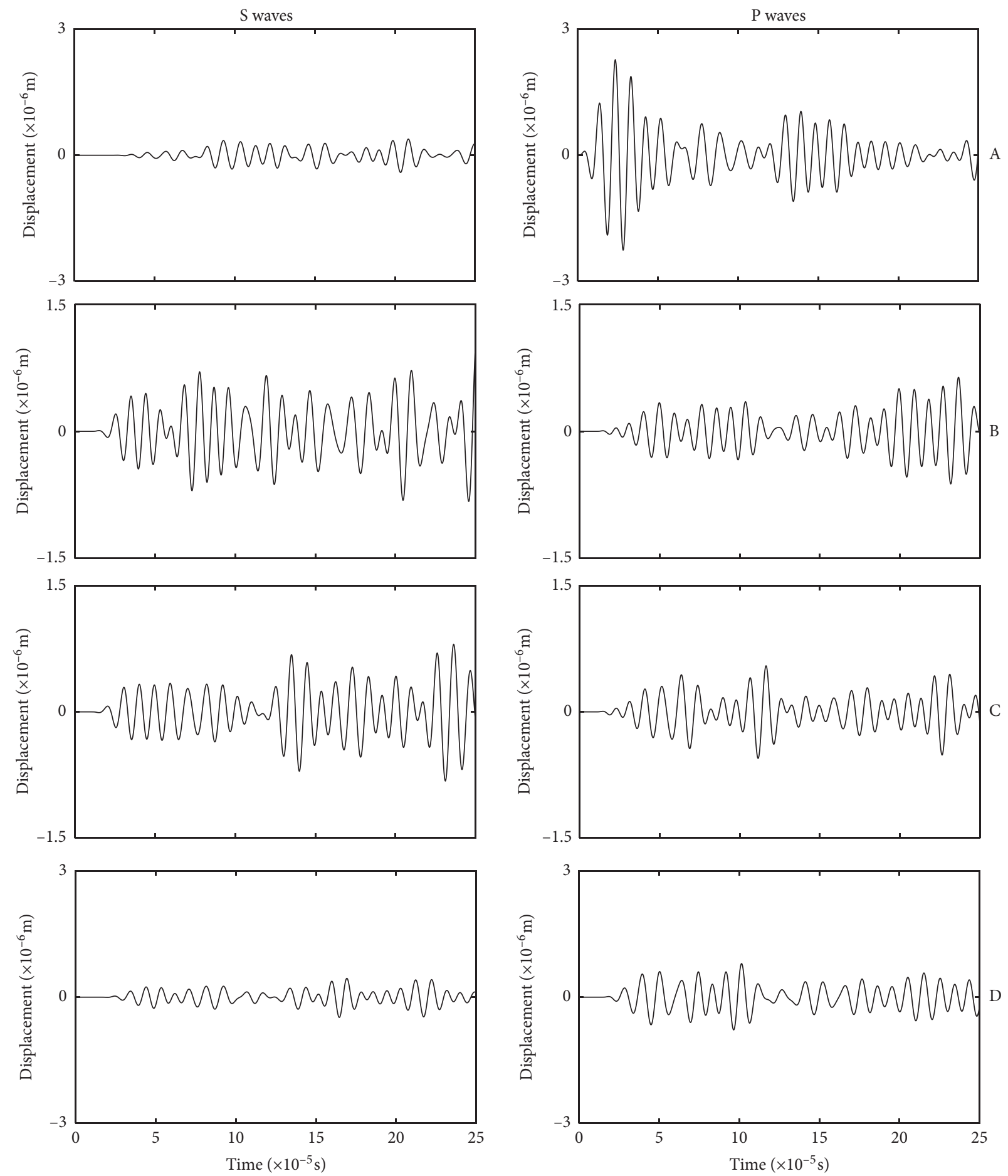

FIGURE 18: Displacement response signals with location 1 in plane. 

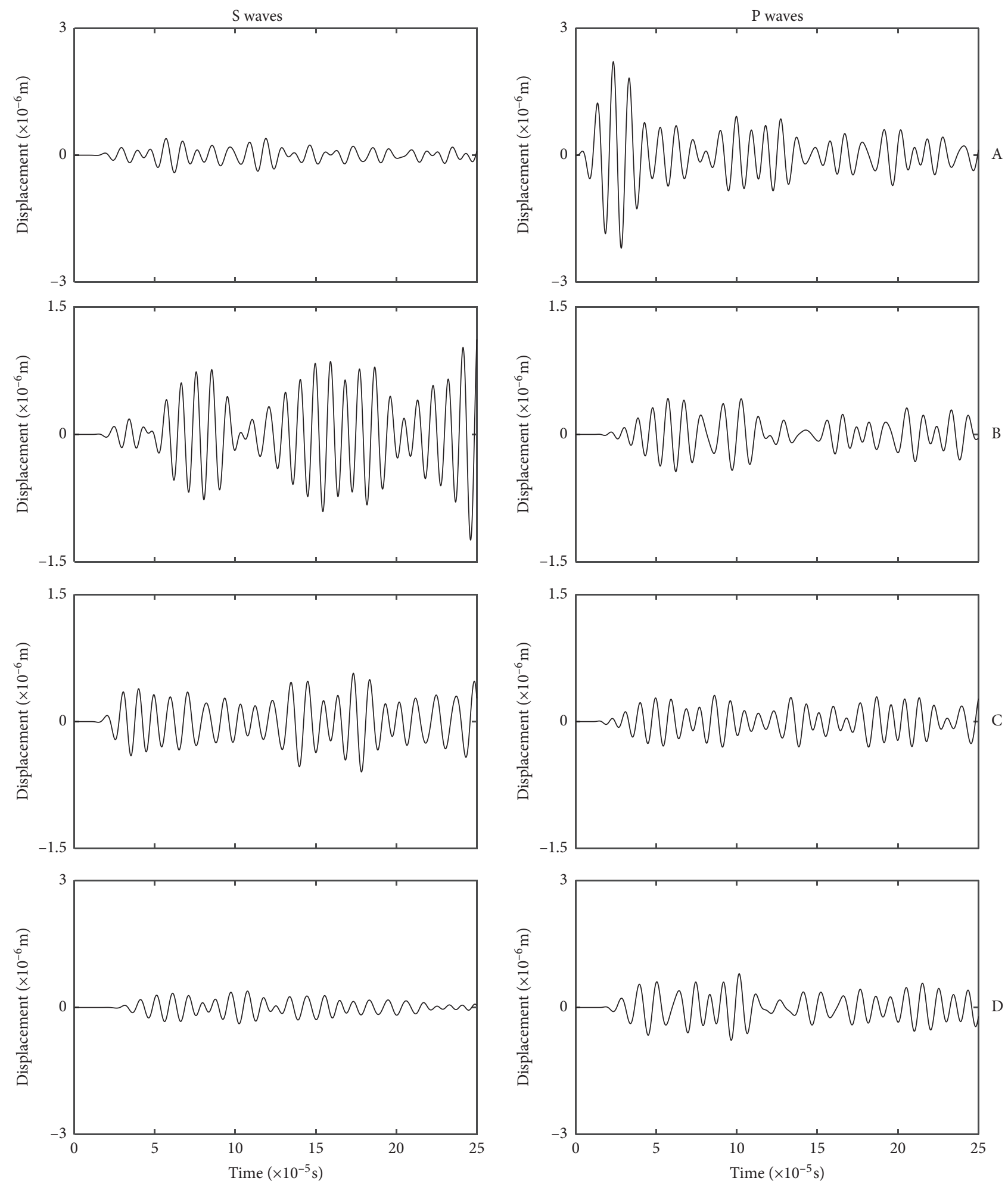

FIGURE 19: Displacement response signals with location 3 in plane.

Hermitian wavelet elements have $20 \times 20$ elements, which have 3,362 degrees of freedom. When the number of Hermitian wavelet elements is further increased, the results of numerical modeling are converged. The numerical results show that the plane structure with crack can be accurately analyzed in this paper. 


\section{Conclusions}

The new plane Hermitian wavelet shape functions are constructed, and the shape functions have been substituted into the finite element equations to calculate the new elements in this paper. The new elements have the characteristics of high precision and less calculation, which can save the calculation time. The new elements are used to analyze the wave motion and to calculate the crack for the different length and location. As the length of crack is increased, the waveform of crack becomes more and more obvious. When the length of crack is consistent, the crack waveform amplitude becomes larger and larger with time development. There are significant effects of wave motion for the crack of different length. As the length of the crack increases, the interval time between the reflected waves and the transmitted waves gets longer and longer. The propagation and reflection of waves will change greatly when the crack location changes. When the crack is not in the middle, the additional displacement response signals can be measured in the $u$ displacement. Due to the influence of the cracks, the waveform and amplitude of waves have changed significantly. This phenomenon can be used to identify the location of different cracks. It is proved that the new elements of researching the wave motion are feasible and effective.

\section{Data Availability}

The data used to support the findings of this research are available from $[3,25]$.

\section{Conflicts of Interest}

The authors declare that there are no conflicts of interest regarding the publication of this paper.

\section{Acknowledgments}

This work was supported by Scientific and Technological Innovation Programs of Higher Education Institutions in Shanxi, the Special Fund for the Doctoral Program in Shanxi (Q2X-2018001), and the Research Fund for the Doctoral Program (YQ-2017021).

\section{References}

[1] B. Khalfi and A. Ross, "Influence of partial constrained layer damping on the bending wave propagation in an impacted viscoelastic sandwich," International Journal of Solids and Structures, vol. 50, no. 25-26, pp. 4133-4144, 2013.

[2] W. Witkowski, M. Rucka, J. Chróścielewski, and K. Wilde, "On some properties of 2D spectral finite elements in problems of wave propagation," Finite Elements in Analysis and Design, vol. 55, pp. 31-41, 2012.

[3] A. Zak, M. Krawczuk, and W. Ostachowicz, "Propagation of in-plane waves in an isotropic panel with a crack," Finite Elements in Analysis and Design, vol. 42, no. 11, pp. 929-941, 2006.

[4] H. Peng, G. Meng, and F. Li, "Modeling of wave propagation in plate structures using three-dimensional spectral element method for damage detection," Journal of Sound and Vibration, vol. 320, no. 4-5, pp. 942-954, 2009.

[5] K. Manktelow, R. K. Narisetti, M. J. Leamy, and M. Ruzzene, "Finite-element based perturbation analysis of wave propagation in nonlinear periodic structures," Mechanical Systems and Signal Processing, vol. 39, no. 1-2, pp. 32-46, 2013.

[6] F. Wang and K. Sett, "Time-domain stochastic finite element simulation of uncertain seismic wave propagation through uncertain heterogeneous solids," Soil Dynamics and Earthquake Engineering, vol. 88, pp. 369-385, 2016.

[7] H. Gravenkamp, S. Natarajan, and W. Dornisch, "On the use of NURBS-based discretizations in the scaled boundary finite element method for wave propagation problems," Computer Methods in Applied Mechanics and Engineering, vol. 315, pp. 867-880, 2017.

[8] M. Komijani and R. Gracie, "An enriched finite element model for wave propagation in fractured media," Finite Elements in Analysis and Design, vol. 125, pp. 14-23, 2017.

[9] A. V. Pamel, G. Sha, S. I. Rokhlin, and M. J. S. Lowe, "Finiteelement modelling of elastic wave propagation and scattering within heterogeneous media," Proceedings of the Royal Society A: Mathematical, Physical and Engineering Science, vol. 473, no. 2197, pp. 1-21, 2017.

[10] A. Stawiarski, M. Barski, and P. Pająk, "Fatigue crack detection and identification by the elastic wave propagation method," Mechanical Systems and Signal Processing, vol. 89, pp. 119-130, 2017.

[11] B. Dubuc, S. Livadiotis, A. Ebrahimkhanlou, and S. Salamone, "Crack-induced guided wave motion and modal excitability in plates using elastodynamic reciprocity," Journal of Sound and Vibration, vol. 476, pp. 1-20, 2020.

[12] M. Komijani, R. Gracie, and Y. Yuan, "Simulation of fracture propagation induced acoustic emission in porous media," Engineering Fracture Mechanics, vol. 229, pp. 1-21, 2020.

[13] M. Gaborit, O. Dazel, P. Göransson, and G. Gabard, "Coupling of finite element and plane waves discontinuous Galerkin methods for time-harmonic problems," International Journal for Numerical Methods in Engineering, vol. 116, no. 7, pp. 487-503, 2018.

[14] C. Renzi, C. Pézerat, and J.-L. Guyader, "Local force identification on flexural plates using reduced finite element models," Computers \& Structures, vol. 144, pp. 75-91, 2014.

[15] N. Nanda, S. Kapuria, and S. Gopalakrishnan, "Spectral finite element based on an efficient layerwise theory for wave propagation analysis of composite and sandwich beams," Journal of Sound and Vibration, vol. 333, no. 14, pp. 31203137, 2014.

[16] Y. Guo, J. Huang, and J. Lai, "A posteriori error analysis of a fully-discrete finite element method for the wave equation," Journal of Computational and Applied Mathematics, vol. 356, pp. 231-247, 2019.

[17] J. S. R. Park, S. W. Cheng, T. Mai, and V. H. Hoang, "Multiscale simulations for upscaled multi-continuum flows," Journal of Computational and Applied Mathematics, vol. 374, pp. 1-26, 2020.

[18] W.-Y. He and W.-X. Ren, "Finite element analysis of beam structures based on trigonometric wavelet," Finite Elements in Analysis and Design, vol. 51, pp. 59-66, 2012.

[19] P. K. Basu, A. B. Jorge, S. Badri, and J. Lin, "Higher-order modeling of continua by finite-element, boundary-element, meshless, and wavelet methods," Computers \& Mathematics with Applications, vol. 46, no. 1, pp. 15-33, 2003.

[20] J. Ma, J. Xue, S. Yang, and Z. He, "A study of the construction and application of a Daubechies wavelet-based beam 
element," Finite Elements in Analysis and Design, vol. 39, no. 10, pp. 965-975, 2003.

[21] X. Chen, S. Yang, J. Ma, and Z. He, "The construction of wavelet finite element and its application," Finite Elements in Analysis and Design, vol. 40, no. 5-6, pp. 541-554, 2004.

[22] J. W. Xiang, X. F. Chen, Z. J. He, and H. B. Dong, "The construction of $1 \mathrm{D}$ wavelet finite elements for structural analysis," Computational Mechanics, vol. 40, no. 2, pp. 325-339, 2007.

[23] X. W. Zhang, X. F. Chen, Z. J. He et al., "The multivariable finite elements based on B-spline wavelet on the interval for 1D structural mechanics," Journal of Vibroengineering, vol. 14, pp. $363-380,2012$.

[24] J. W. Xiang, Y. X. Wang, Z. S. Jiang et al., "Numerical simulation of plane crack using hermite cubic spline wavelet," Computer Modeling in Engineering \& Sciences (CMES), vol. 88, pp. 1-16, 2012.

[25] X. Xue, X. Chen, X. Zhang, and B. Qiao, "Hermitian plane wavelet finite element method: wave propagation and load identification," Computers \& Mathematics with Applications, vol. 72, no. 12, pp. 2920-2942, 2016.

[26] X. Xue, X. Zhang, B. Li, B. Qiao, and X. Chen, "Modified Hermitian cubic spline wavelet on interval finite element for wave propagation and load identification," Finite Elements in Analysis and Design, vol. 91, pp. 48-58, 2014. 